\title{
The effect of movement velocity on form perception: Geometric illusions in dynamic displays
}

\author{
PAOLO VIVIANI and NATALE STUCCHI \\ University of Geneva, Geneva, Switzerland
}

\begin{abstract}
We investigated the effects of movement velocity on the perception of simple geometric trajectories. We show that when an ellipse is traced by the continuous displacement of a spot against an empty background, the subjective aspect ratio $(R=$ vertical axis/horizontal axis) of the figure depends on the law of motion of the spot. If the tangential velocity of the spot is constant, very large and subject-specific biases emerge in the perception of the aspect ratio. If the tangential velocity of the spot is made equal to that of an elliptic motion with aspect ratio $R<1$, and resulting from the vectorial composition of two harmonic functions (Lissajous motion), there is a general trend to perceive the ellipse as being flatter than in reality. The effect, however, is not symmetric: when the velocity follows a Lissajous modulation with $R>1$, highly significant biases are still present in most subjects, but no common trend emerges from the experimental population. The results are discussed in the context of recent findings on the relationship between form and kinematics in spontaneous human movements.
\end{abstract}

The term "visual illusion" encompasses all those phenomena in which perception belies objective reality. However, a distinction is generally drawn between two large classes of illusions (cf. Coren \& Girgus, 1978a; Robinson, 1972). The best known and historically well-established phenomena occur in the perception of static configurations (the so-called geometric illusions, e.g., the MüllerLyer double arrow). Although no single theory exists to account for all specific cases, it is generally agreed that the illusory effect arises naturally from the interaction of a specific visual configuration with the basic processes of vision. As Gibson (1966) put it, "we should never have expected equal lengths to appear equal when they are incorporated in different figures" (p. 313). In particular, a plausible line of reasoning, supported by some experimental evidence (e.g., Gillam, 1971), suggests that most geometric illusions result from the tridimensional interpretation of planar configurations. Normally, the basic preconception that real objects do not change size as they move about overrides the disparities of retinal sizes and assures a veridical perception in 3-D space. Geometric distortions would then occur because the same compensatory mechanism is inescapably applied also to 2-D visual stimuli.

The second group of phenomena involves movement and is quite heterogeneous. Broadly speaking, illusions of this type make us perceive a movement that is different from the objective one (as in the wheel illusion, or in the dynamic configurational effects studied by Johansson,

This work was supported by the National Research Council of Italy and by FNRS (Switzerland) Research Grant 1.150.0.85. Correspondence may be addressed to Paolo Viviani, Faculty of Psychology and Educational Sciences, University of Geneva, 24 Rue de Général Dufour, 1211 Genève 4, Switzerland. 1950a, 1950b, 1973, 1977, and Restle, 1979), or even a movement when there is none, as in the "phi-movement" (Benussi, 1912; Korte, 1915; Wertheimer, 1912) and "sigma-movement" (Adler \& Grüsser, 1979; Behrens \& Grüsser, 1978; Lamontagne, 1973) effects. Some of these phenomena-such as the phi-movement-are likely to be rooted in the neurophysiological hardware of our visual system (see Grüsser \& Grüsser-Cornhels, 1973). By contrast, other, more complex effects-such as the forceful perception of rigid or even elastic motions induced by the coordinated displacement of a few light points-are suggestive of the involvement of cognitive, "hypothesismaking" processes beyond the visual system (Johansson, 1978). Specifically, an underlying cause for these complex dynamic effects may again be the implicit assumption that the actual stimulus is the 2-D projection of a 3-D configuration (Börjesson \& von Hofsten, 1972, 1973; Braunstein \& Andersen, 1984).

In this report, we document a novel type of visual illusion involving movement. It will be argued that, also in this case, the illusory effect hinges on preconceptions lying beyond the purely visual domain. However, unlike the examples mentioned above, the illusions manifest themselves as movement-induced distortions of a geometric form. The motivation for the work reported here originates from previous and ongoing research on the structural properties of human hand movements. It has been shown that, in drawing plane trajectories, humans spontaneously comply with a general principle of covariance relating the law of motion of the movement to the differential geometric properties of the trajectory (Lacquaniti, Terzuolo, \& Viviani, 1983; Viviani \& Cenzato, 1985; Viviani \& Terzuolo, 1982). Specifically, it has been demonstrated that, at all points of the trajectory sufficiently removed from a point of inflection, the tangential 
velocity $V$ of a drawing movement is proportional to the cubic root of the instantaneous radius of curvature $R$. In the case of closed trajectories that do not contain inflections, the relationship is extremely tight.

This empirical finding has some implications concerning the mode of production that humans follow spontaneously when they trace these closed figures. The geometric trajectory of a movement can be described either by an explicit relationship, $y=f(x)$, between the coordinates of its points, or by the so-called parametric form, $x=x(t)$; $y=y(t)$, in which the time course of the Cartesian components of the movement is independently specified. The correspondence between the two forms of description is not one-to-one. A given pair of time-varying Cartesian components, $x(t)$ and $y(t)$, defines uniquely the trajectory of the corresponding movement, but one trajectory may result from the vectorial sum of infinitely many pairs of components. However, to each pair of components, there corresponds a unique function, $l=l(t)$, describing the time course of the curvilinear coordinate and, consequently, a unique time course of the tangential velocity, $V(t)=d l / d t$. Conversely, if one specifies the geometric trajectory and the tangential-velocity function, then the Cartesian components are uniquely identified. Thus, if the tangential velocity of drawing movements were unconstrained, there would be no principled criterion for selecting one specific pair of components to trace a given form. The empirical fact that we actually do constrain the tangential velocity to be a function of the radius of curvature means instead that only one well-defined pair of components is consistently selected which depends on the form of the movement (actually, the components are only specified up to a scaling factor that accounts for the possibility of choosing the average velocity of the movement). It can be shown mathematically (Viviani \& Cenzato, 1985) that, when the form to be traced is an ellipse, the $V-R$ relationship implies that the Cartesian components must be harmonic functions with the same frequency. For historical reasons, this mode of production, which humans select spontaneously when drawing ellipses, has been dubbed the Lissajous model of trajectory formation.

In recent experiments on visuo-manual tracking (Viviani, Campadelli, \& Mounoud, 1987; Viviani \& Mounoud, 1988), we investigated the effects on the motor response of using visual targets whose law of motion violated in a systematic way the covariation between velocity and radius of curvature which is observed in spontaneous drawing movements. In particular, quite consistent distortions occurred in the pursuit of elliptic trajectories when the law of motion imposed on the target was not the one predicted by the Lissajous model for the ellipse being pursued, but rather the one that was predicted for the same ellipse rotated by $90 \%$. It can be suspected that these distortions are the manifestation of a conflict between the intrinsic properties of the motor systems embodied in the Lissajous model and the characteristics of the driving visual input. However, by considering only the motor output, as we did in the research mentioned above, one can- not rule out the hypothesis that, at least in part, the effect arises at the perceptual level. The experiments described in this report were designed to ascertain whether the perception of dynamic ellipses is influenced by the specific law of motion used to trace the outline of the pattern. In particular, we considered the discrimination of the ellipse aspect ratio $\left(R_{g}=\right.$ vertical axis/horizontal axis) with an adaptive version of the yes-no paradigm. This discrimination task has already been investigated in static conditions (Sleight \& Austin, 1952; Sleight \& Mowbray, 1951) to determine whether the well-known horizontal-vertical illusion generalizes to the perception of geometric shapes. Although the conclusions of these studies are not totally unambiguous, they provide an appropriate reference for discussing the results of the dynamic experiment reported here.

\section{METHOD}

\section{Subjects}

Twenty-three subjects participated in the experiments. All but 4 of them were psychology students in their early twenties. The others were professional colleagues. All had normal or correctedto-normal vision. The results from 3 subjects had to be discarded because they were obviously not capable either of complying with the assignment or of maintaining the required concentration throughout the experiment. The subjects were naive as to the purpose of the experiments and the nature of the difference between conditions. After the experiments, some subjects reported spontaneously that they had noticed the systematic modulation of the tangential velocity in two experimental conditions. Since these modulations were large (see below), it is likely that all subjects had actually been aware of them.

\section{Apparatus}

The subject sat in front of an X-Y monitor (Tektronix 564 with a T564-200 green phosphorous). A pyramidal viewer of black matt plastic, similar to that used for adapting Polaroid cameras, was mounted on the monitor screen to prevent the subject from seeing the environment, which was kept in dim light. The moving spot was the only visible light and no spatial reference was available in the visual field. A chinrest was fitted to the lower edge of the viewer to prevent fatigue and to minimize head movements. Responses were recorded with two light-pressure microswitches (marked "Flat" and "Tall"), which the subjects operated independently with their left and right index fingers, respectively.

\section{Stimuli and Task}

Stimuli were fed in the $\mathrm{X}-\mathrm{Y}$ amplifiers of the oscilloscope by the computer interface. In all cases, the beam on the screen traced elliptic patterns of various eccentricities in a counterclockwise direction. The brightness of the beam was about $6 \mathrm{fL}$. The perimeter of the ellipses was kept constant throughout all experiments: $Q=13.2 \mathrm{~cm}$. Because of the persistence of the screen, about one tenth of the complete trajectory was visible at any one time. At the average viewing distance imposed by the chinrest $(33 \mathrm{~cm})$, the most elongated ellipses encompassed $9.8^{\circ}$ of visual angle. In a single presentation, the entire figure was traced 10 times at an average rate of $.6 \mathrm{sec}$ per cycle. Let $A_{x q}$ and $A_{y g}$ denote the horizontal and vertical axes of the ellipse, respectively. The aspect ratio is defined as $R=A_{y g} / A_{x g}$. The major axis of the figure could be either horizontal $\left(R_{g} \leq 1\right)$ or vertical $\left(R_{g} \geq 1\right)$. In the first case, the eccentricity, $\Sigma$, is defined as $\left[1-\left(A_{y g} / A_{x g}\right)^{2}\right]^{1 / 2}$; in the second case, it is defined as $\left[1-\left(A_{x g} / A_{y g}\right)^{2}\right]^{1 / 2}$. Throughout this paper, the two pos- 
sible orientations of the figure are distinguished by the sign of the eccentricity, $\Sigma$, which is conventionally set to be positive when $R_{g} \leq 1$ and negative when $R_{g} \geq 1$. At the end of the 10 cycles, the screen was left blank and the subject had to indicate, with the appropriate response key, whether he/she thought that the direction of the major axis of the ellipse was horizontal (response "'flat") or vertical (response "tall"). No time constraints were imposed, but the subjects were encouraged to rely exclusively on their immediate visual impression. In fact, most subjects reported that a decision was sometimes made even before the end of the presentation. Responses were followed by a minimum intertrial interval of $5 \mathrm{sec}$, after which a 500 -cps tone signaled to the subject that he/she could trigger the next stimulus by pressing either of the two response keys. An experimental session was composed of a sequence of stimulus-response pairs (trials) within which both the eccentricity and the orientation of the major axis changed independently. Sessions ended when a fixed criterion was met (see below), and generally comprised between 45 and 80 trials. On the average, a session lasted $20 \mathrm{~min}$. Changes in the geometric parameters of the stimuli were scheduled by the following algorithm. The controlling program had in store two sequences of 29 stimuli, one starting with an eccentricity $\Sigma_{\mathrm{g}}=+.70$ and decreasing by steps of .05 to a final value of $\Sigma_{g}=-.70$, and the other ascending from $\Sigma_{g}=-.70$ to $\Sigma_{g}=+.70$, in steps of .05 . The stimuli to be presented were identified by pointers moving along the sequences and by a random selection of one of the two sequence. On the first trial, the selection probability was .5 . On any successive trial $k$, the probability of selecting one sequence exceeded .5 by an amount which was an increasing function of the relative proportion of stimuli already taken from the other sequence in the preceding $k-1$ trials. This selfadjusting scheme effectively prevented the occurrence of long sequences of one stimulus type and assured a stricter balance between the two orientations than the one expected from a purely bernouillian scheme. At the beginning of the experiment, both pointers were on the first position. On any trial thereafter, if the answer was correct, the pointer in the sequence from which the stimulus had been drawn moved one step toward the middle (15th) position which, in both sequences, corresponded to a circular pattern $\left(\Sigma_{g}=0\right)$. If the answer was wrong, the pointer moved one step away from the middle position (see Figure 1). If the pointer was already in the middle position, there was no correct answer and the pointer could only move away from it. The consistency of this procedure was assured by the fact that extreme values of eccentricity $\left(\Sigma_{g}= \pm .70\right)$ never gave rise to wrong answers. The controlling program kept a record of the number of times that the direction of pointer movement along the sequences was inverted. The experiment ended when at least 10 inversions had been recorded in each sequence. The three left panels in Figure 1 display typical examples of the sequence of stimuli presented to 1 subject in different experiments. All subjects participated in three experimental sessions which, in some cases, were concentrated in a single day and, in other cases, took place on different days. In every session, the geometric parameters of the stimuli and the experimental procedure were those described above. The only difference concerned the law of motion of the spot that traced the trajectories. In Experiment 1 (Panel A), the tangential velocity of the spot was constant throughout the trajectory. In Experiment 2 (Panel B), the tangential velocity at any point of the trajectory was equal to that of an elliptic Lissajous movement with eccentricity $\Sigma_{d}=+.90$. In Experiment 3 (Panel C), the tangential velocity was equal to that of an elliptic Lissajous movement with eccentricity $\Sigma_{d}=-.90$. The same kinematics was imposed on all stimuli within a session. The order in which the three conditions were administered was randomized across subjects. The right panels in Figure 1 illustrate the relationship between the geometry of the trajectories and the corresponding velocity in the three conditions.
A formal definition of these kinematic conditions is provided in the Appendix.

\section{RESULTS}

Because of the response-contingent paradigm adopted for the experiments, the values $\Sigma_{g i}$ of the eccentricity in the sequences of stimuli presented in each session afford a complete description of the results. To characterize the perceptual performances of the subjects, we performed two types of analysis on these raw data. The first analysis took into account only the values $\Sigma_{g i}$ for the last 10 inversions in each sequence (each inversion corresponds to a wrong answer, see Method section). The differential limen $\Sigma_{0}$ (i.e., the eccentricity of the figure that is subjectively perceived as a circle) was estimated by the average of these 20 values of $\Sigma_{g i}$. The arithmetic means and standard deviations of $\Sigma_{g i}$ for each subject in the three experimental conditions are reported in Table 1. A twoway analysis of variance ( 20 subjects $\times$ three conditions; 20 replicates per cell) indicated significant effects of the two factors as well as a significant interaction. As shown in column A of Table 1, the very fact that patterns were traced dynamically induced very large, consistent, and subject-specific biases in the perception of the aspect ratio of the figures. In particular, what was perceived as a circle could actually be either a vertically or a horizontally oriented ellipse. For many subjects, the eccentricity of this ellipse would clearly be detected if the patterns had been presented statistically (Sleight \& Austin, 1952).

In most cases, modulating the velocity with which the trajectories were traced induced a shift of the differential limen (see Table 1, columns B and C). However, the consistency of the individual performances remained quite high. The overall behavior of the experimental population in the three dynamic conditions is contrasted in Panels A, B, and C of Figure 2. Panel A shows the cumulative probability function of the inversion values, $\Sigma_{g i}$, in the constant-velocity condition. The function was calculated by pooling the data of all subjects ( 400 values). In agreement with the results of Table 1, the median of the distribution is close to zero. Thus, no definite trend emerges in the direction of the individual biases. Although the variance of the distribution includes both the individual variabilities and the difference across subjects, the mixture is still closely approximated by a cumulative Gaussian ogive. The specific influence of the velocity modulation revealed by the analysis of variance can be described by comparing individual performances in each test condition with the corresponding performances in the constantvelocity experiment. The middle (B) and right (C) panels in Figure 2 show the cumulative probability function of the difference between the values of $\Sigma_{g i}$ for the indicated modulations and the values of $\Sigma_{g i}$ in the control. Differences were calculated on pairs of inversions having the same ordinal position in the response sequences. This pair- 

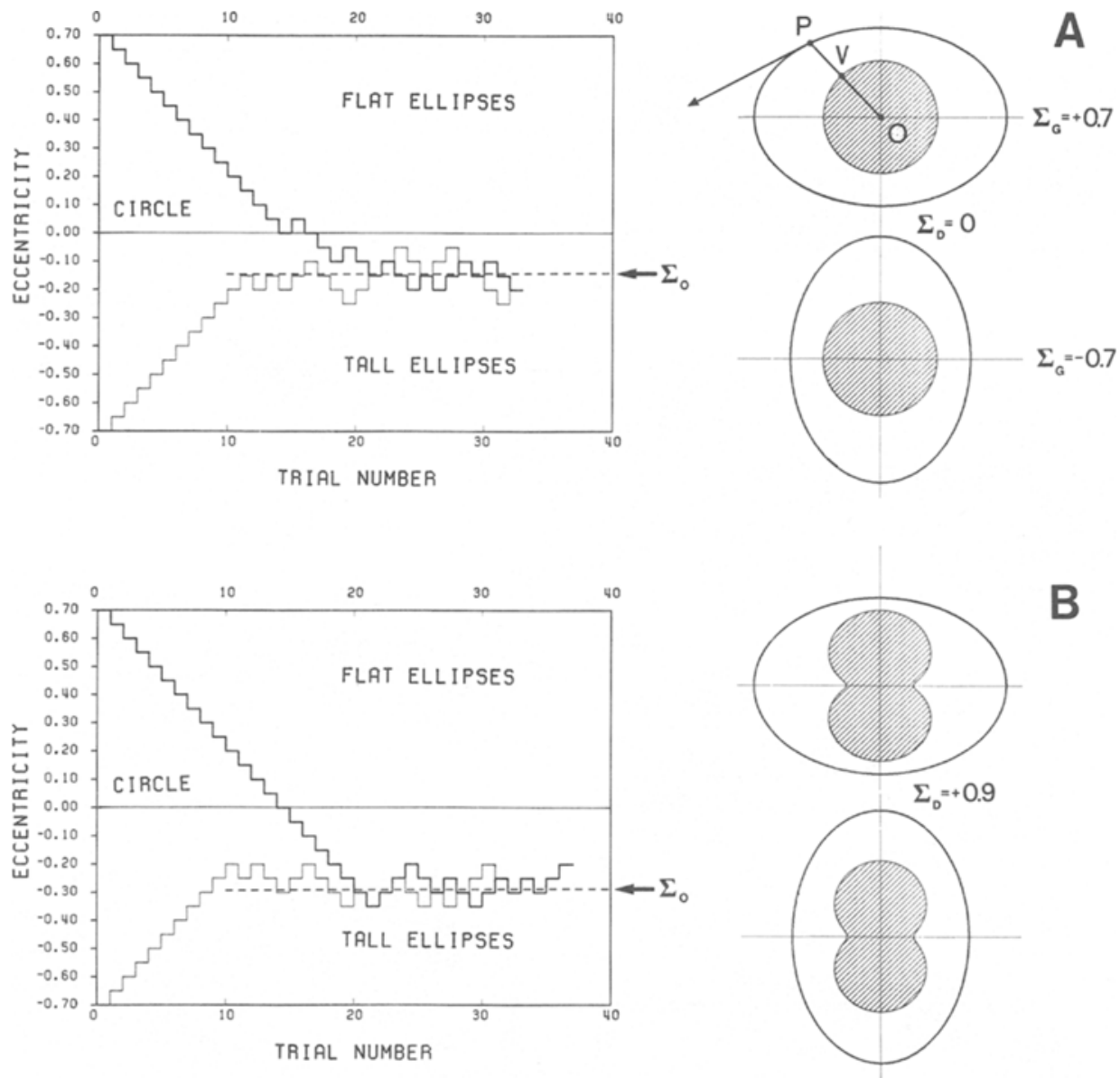

B
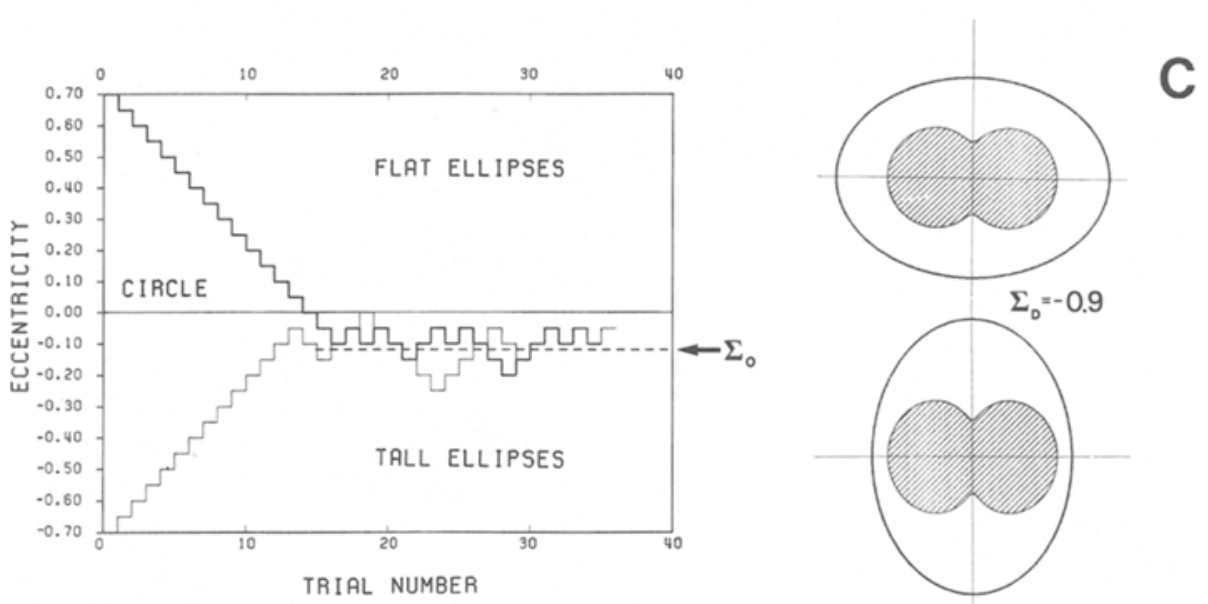

Figure 1. Experimental conditions and typical trials. On the right, the outlines of the two extreme stimuli $\left(\Sigma_{z}= \pm .70\right)$ illustrate the relationship between geometry and kinematics in the three experimental conditions $(A, B$, and $C)$. The tangential velocity at any point $P$ of the trajectory is proportional to the distance $V O$ defined by the cross-hatched polar diagrams. Note that the actual time course of the tangential velocity does not have the discontinuities that may be suggested by the polar representation. Formally, the polar diagrams represent the velocity of Lissajous elliptic movements with eccentricity $\Sigma_{d}=0.0(A),+0.90$ (B), and -0.90 (C) and with the same perimeter of the geometric ellipses (see Appendix). All stimuli within an experimental condition have the same velocity profile. The three left panels illustrate the complete experimental history of 1 subject. Stimuli in successive trials are randomly selected from two sequences. Trial numbers on the abscissa are relative to each sequence separately. Starting from the two extreme stimuli, the eccentricities are reduced after a correct answer and increased after a wrong answer. The experiment ends after a fixed number of inversions. The differential limen $\Sigma_{0}$ (arrows) is defined as the mean eccentricity of the last $\mathbf{1 0}$ inversions in both sequences. The small variability of the response sequences is typical of what was observed in most trials. 
Table 1

Individual Estimates of the Differential Limen $\Sigma_{0}$

\begin{tabular}{|c|c|c|c|c|c|c|}
\hline \multirow[b]{3}{*}{ Subject } & \multicolumn{6}{|c|}{ Conditions } \\
\hline & \multicolumn{2}{|c|}{$\mathrm{A}\left(\Sigma_{d}=0.0\right)$} & \multicolumn{2}{|c|}{$\mathrm{B}\left(\Sigma_{d}=+.90\right)$} & \multicolumn{2}{|c|}{$\mathrm{C}\left(\Sigma_{d}=-.90\right)$} \\
\hline & $M$ & $S D$ & $M$ & $S D$ & $M$ & $S D$ \\
\hline 1 & .012 & .095 & -.210 & $* \quad .101$ & -.127 & * .062 \\
\hline 2 & .025 & .099 & -.245 & * .050 & -.057 & * .079 \\
\hline 3 & .140 & * . 125 & -.237 & * .082 & .265 & * .076 \\
\hline 4 & .232 & * .058 & .092 & $* \quad .111$ & .125 & * .096 \\
\hline 5 & .007 & .079 & -.095 & * .079 & .045 & * .065 \\
\hline 6 & .310 & * .058 & .072 & * . .098 & .167 & * .069 \\
\hline 7 & .102 & $* \quad .071$ & -.127 & $* \quad .100$ & .007 & .101 \\
\hline 8 & .282 & * .050 & -.157 & * $\quad .051$ & .230 & $* \quad .075$ \\
\hline 9 & -.015 & .092 & -.020 & .048 & .032 & .086 \\
\hline 10 & .165 & * . 106 & .157 & $* \quad .051$ & .062 & * .083 \\
\hline 11 & .032 & .096 & .195 & $* \quad .067$ & .157 & * .069 \\
\hline 12 & .092 & * .078 & .075 & * .084 & -.042 & .126 \\
\hline 13 & -.022 & $* \quad .029$ & .062 & * .052 & .000 & .042 \\
\hline 14 & -.220 & * .083 & -.052 & .187 & -.217 & * $\quad .069$ \\
\hline 15 & .130 & * .074 & .232 & * .055 & .175 & * .062 \\
\hline 16 & -.152 & * $\quad .077$ & -.190 & * .056 & .007 & .078 \\
\hline 17 & -.412 & * . .063 & -.115 & * .110 & -.220 & * .097 \\
\hline 18 & -.130 & * $\quad .087$ & -.090 & * .083 & -.032 & .066 \\
\hline 19 & -.200 & * $\quad .074$ & -.217 & * . 051 & -.065 & * $\quad .077$ \\
\hline 20 & -.125 & $* \quad .078$ & -.285 & * .053 & -.090 & * .058 \\
\hline $\begin{array}{l}\text { Av. } \\
S D\end{array}$ & $\begin{array}{l}.013 \\
.172\end{array}$ & & $\begin{array}{r}-.058 \\
.153\end{array}$ & & $\begin{array}{l}.021 \\
.132\end{array}$ & \\
\hline
\end{tabular}

Note-Averages and standard deviations of the individual limina are reported. Significant differences exist among subjects $[F(19,1140)=$ $143.09, p<.001]$ and among experimental conditions $[F(2,1140)=$ $105.93, p<.001]$. The interaction is also significant $[F(38,1140)=$ $34.96, p<.001]$. A significant correlation exists between the individual limina in Conditions $A$ and $C\left(r_{A C}=+.801\right)$. Correlations between the limina in Condition $\mathbf{B}$ and those in both Condition $\mathbf{A}$ and Condition B are much weaker $\left(r_{A B}=+.378 ; r_{C B}=+.373\right) . \quad * \Sigma_{0}$ is significantly different from zero.

ing is somewhat arbitrary, but it does not bias the results because sequences in different experiments were statistically independent. Moreover, it permits a compensation for the fact that inversions early in the sequence generally occurred for eccentricities that were higher than the limen.

The distribution in Panel B demonstrates that when the tangential velocity of the spot was modulated as shown diagrammatically inset, the resulting shift of the differential limen was mostly in one direction. Irrespective of the eccentricity and orientation of the ellipse that was perceived as a circle in the control situation, the majority of the subjects experienced an illusory contraction of the vertical direction. The effect of the spot kinematics was different in the second test condition, in which the orientation of the velocity modulation was rotated by $90^{\circ}$ (Panel C). The biasing action demonstrated in Panel B disappears (the median of the distribution is not significantly different from zero) but, surprisingly, the variability among individuals is reduced with respect to both the control and the first test condition.

The differential sensitivity of each subject in each condition can be estimated from the standard deviations of the limina reported in Table 1 . Individual performances were too different to warrant the use of the probability distributions in Panels A, B, and C of Figure 2 for estimating the differential sensitivities of the entire population. A second type of analysis was devised to this end. It consisted of calculating the psychometric functions for the aspect ratio using all the available answers. Classically, the psychometric function would be calculated by estimating the probability of a response "Flat" for each possible value of the eccentricity. However, to be able to pool the results of all subjects, the probabilities were in fact estimated after subtracting from all eccentricities the individual limina $\Sigma_{0}$ in the constant-velocity condition (Table 1, column A). All data points with probabilities greater than .995 and less than .005 were censored to avoid outlier effects in the linear-regression analysis. Finally, the data were scaled by the $z$ transformation. The results for each condition are shown in the lower panels of Figure 2. The least square estimates of $\Sigma_{0}$ (average) refine the estimates previously obtained from the analysis of the points of inversion. In particular, the $95 \%$ confidence band around the regression lines demonstrates the statistical significance of the general biasing effect when $\Sigma_{d}=+.90$. Finally, the desired estimate of the differential sensitivity of the entire population was calculated as the inverse of the slope of the regression line through the $z$ values (labeled "ST. DEV." in the figure; the higher the value of the standard deviation, the lower the sensitivity). Statistical analysis showed that, after removing the spurious effects of individual differences, a significant difference remained in the differential sensitivity for the three conditions ( $p<.01$ for all pairwise comparisons).

\section{DISCUSSION}

Consistent distortions of the aspect ratio occur when a simple geometric form is displayed dynamically. A comparison of the results in the three experimental conditions suggests the possibility of distinguishing two types of distortion. The first seems to be contingent on the very presence of movement. Its direction and magnitude vary from subject to subject, but the effect is significant in all but a few individuals. The second type of distortion is related to the specific law of motion with which the form is traced, and interacts in a complex way with the first effect. We begin by comparing the results in the constantvelocity experiment to those of analogous experiments involving static displays. The available literature on the discrimination of the aspect ratio focuses on just two shapes: rectangles and ellipses. In both cases, the experiments were designed to verify the hypothesis that the classical horizontal-vertical illusion carries over to the perception of 2-D figures. In the case of rectangles, the pattern of results is complex. Veniar (1948) observed only in some subjects a tendency to perceive rectangles as more elongated in the vertical direction. When present, the magnitude of the constant error was about the same (6\%) as that measured in the inverted- $T$ form of the classical illusion (7.2\%; Finger \& Spelt, 1947). Moreover, differential sensitivity to squareness (Weber ratio $=.014$ ) compares well 

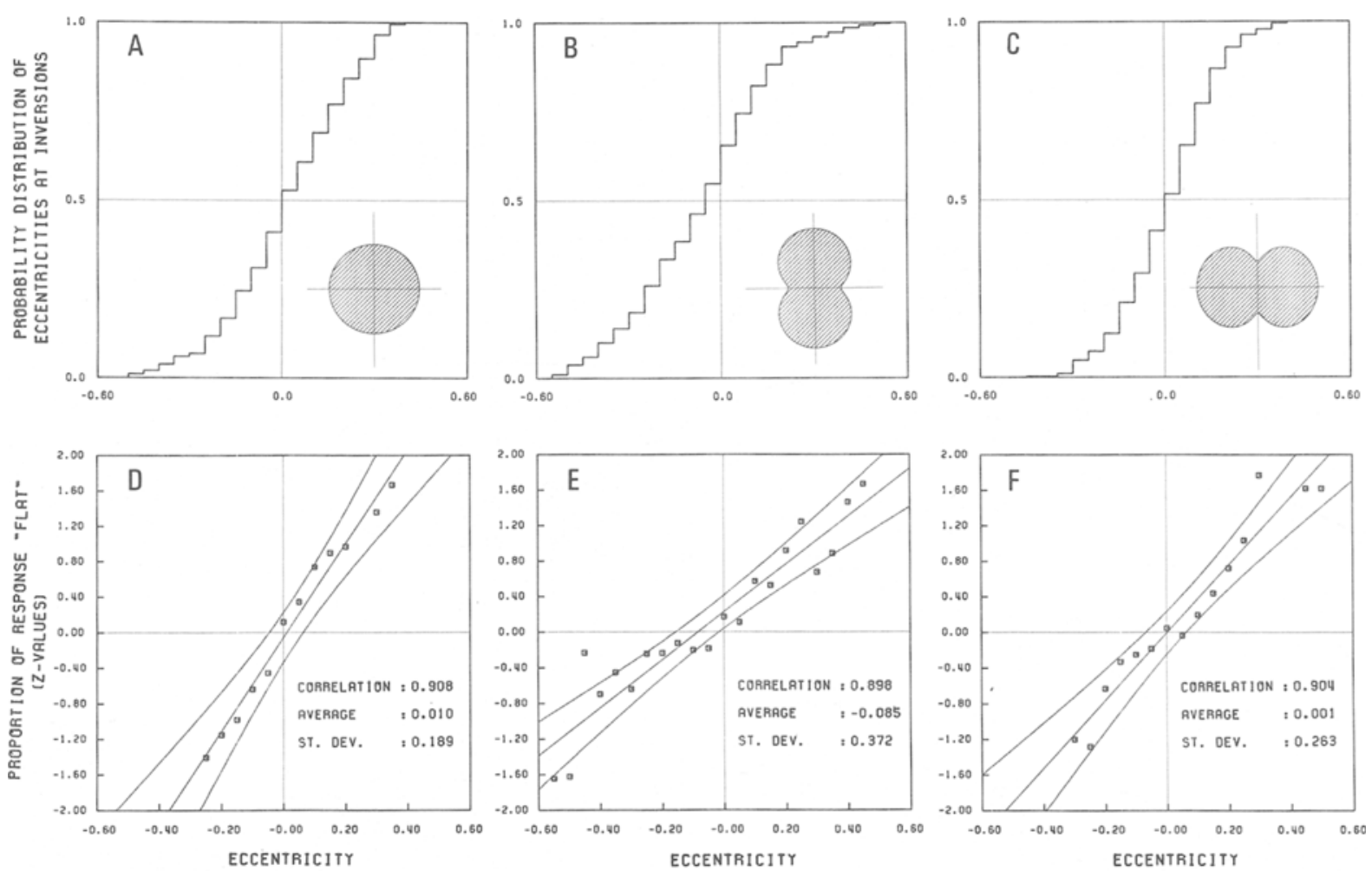

Figure 2. Differential sensitivity of the aspect ratio. Cross-hatched polar diagrams shown inset identify the constant-velocity condition (Panels $A$ and D: $\Sigma_{d}=0.0$ ) and the two conditions with variable velocities (Panels B and E: $\Sigma_{d}=+.90$; Panels $C$ and F: $\Sigma_{d}=-.90$ ). Panel A: Probability distribution (PDF) of the eccentricities at which a wrong answer was given (inversions). Panels B and C: PDFs of the difference between the eccentricities at inversion in the indicated conditions and those in the constant-velocity condition. Each PDF was calculated by pooling the results of all subjects ( 400 values). Despite large individual differences (see Table 1), the PDFs are approximated reasonably well by normal ogives. Panels D, E, and F: Psychometric functions ( $z$ values) obtained by subtracting from the individual results in each condition the differential limen $\Sigma_{0}$ measured in each subject in the constant-velocity condition. Constant errors (average) and JNDs (SD) for the entire experimental population are estimated from the linear regression through the data points. Confidence hyperbolas at the .95 level are also indicated.

with that for line length (.01-.02; Ladd \& Woodworth, 1911 , p. 371). In the study by Sleight and Austin (1952), fewer than half of their subjects overestimated the vertical side of the rectangles, whereas the others experienced the opposite illusion. In a more complex experimental situation (Sleight \& Mowbray, 1951), overestimation of the horizontal dimension appeared to be the rule.

As for ellipses, the study by Sleight and Austin (1952) provides a consistent picture of the performance in the static condition and an appropriate basis for discussing the dynamic case. We begin by noting that, when the values $\Sigma_{0}$, which corresponds to a subjective impression of circularity, are translated in terms of the ratio between minor and major axes, the illusory effect may appear very small indeed. Misjudgments in most classical geometric illusions exceed $10 \%$ and may even attain $20 \%$ to $25 \%$ (Coren \& Girgus, 1978b). In contrast, consider for instance Subject 19 of the present study (Table 1A): the ellipse that he perceived as a circle $\left(\Sigma_{0}=-.200\right)$ had an aspect ratio of .98 , a mere $2 \%$ deviation from reality. In fact, though, the illusory effect is of the same order of magnitude as the one reported by Sleight and Austin for static ellipses. If one converts the three-choice response probabilities reported by those authors (Table 4, p. 284) in terms of psychometric functions, the results $\left(\Sigma_{0}=.143\right.$; JND $=.121)$ are indeed similar to those in Table 1 and Figure 2D. The major qualitative difference between the two studies is that in the static case all subjects experienced an illusory stretching in the vertical direction, whereas in the presence of movement the illusion could occur in either direction. Since in our experiment the algebraic mean of the limina across subjects is close to zero (cf. Table 1A), it could appear that the net effect of movement is to neutralize the horizontal-vertical illusion. However, in both the static and the dynamic condition, most individual JNDs were small, the corresponding biases were highly significant, and the striking self-consistency of the responses only enhances the quantitative differences among individuals. Thus, although no compelling hypothesis can be offered to explain the perceptual biases, we are inclined to suppose the existence of a specific effect related to movement that is independent of the horizontalvertical illusion. Moreover, two general suggestions can be put forward. 
First, we believe that, in the present case, the involvement of an illusory perception of depth-which has often been cited as a factor underlying geometric illusionsshould be ruled out. As demonstrated by Börjesson and von Hofsten (1972, 1973), a necessary condition for perceiving motion in depth is the presence of relative motion between individual components of the visual display, something that could never arise in our experimental conditions. In fact, our subjects never reported the compelling impression of depth that can be elicited by the concurrent motion of two or more dots.

Second, short-term memory mechanisms are likely to be involved in the genesis of the illusion. The argument goes as follows. As described in the Method section, the combination of tracing speed and screen persistence was such that only a small fraction of the total outline of the ellipse was visible at any one time. When several trajectories are displayed simultaneously, even comparably small fractions of the total dynamic event may be sufficient to detect some of its relational properties, such as the presence of rigidity constraints (Todd, 1982). By contrast, it is difficult to see how a global metric property of the ellipse-and in particular the aspect ratio-could be computed from isolated segments of trajectory that, as in our case, do not even encompass one fourth of the total outline. Eye movements were not restrained and were not measured. However, at an average speed of $22 \mathrm{~cm} / \mathrm{sec}$ (see Method section) along a curved path, smooth pursuit movements could not have a significant role as has been suggested for discrimination of linear velocity (Runeson, 1974). Moreover, gauging distances through eye saccades (cf. Coren, 1981) was also impossible because the visible trace was smaller than the distance between two successive extrema of the curvature. In conclusion, decisions were to be arrived at by integrating partial samples of the stimulus, none of which, per se, carries sufficient discriminal information on the aspect ratio of the pattern. Since figural information is supplied through a time-limited window, all spatial relationships among elements lying outside the window span must be recoded into temporal relationships. Thus, the distortions of subjective circularity observed in the constant-velocity condition may be construed as the consequence of highly consistent and subject-specific limitations in the process of temporal integration.

Within this general framework one can entertain the hypothesis that the large individual biases result from an improper assignment of the horizontal and vertical directions. In fact, the deviation from circularity can be construed as the ratio of two perceived distances: that between the uppermost and lowermost portions of the figure and that between the left-most and right-most portions. If the perceived relative position of the up-down and left-right orientations is altered by the process of temporal integration, the subjective aspect ratio could also be altered. The old observation that the assignment of spatial orientations is indeed affected by movement (Benussi, 1918) lends circumstantial support to this hypothesis.
We come now to consider the illusory effect that is specifically related to the modulation of the tangential velocity. In both test conditions, most subjects exhibited a significant perceptual bias (Table 1, Panels B and C). However, in only the first condition did a coherent trend emerge from the individual performances. In fact, 14 out of 20 subjects changed significantly $(p \leq .05)$ the point of subjective circularity, going from the constant-velocity condition (Table 1A) to the condition $\Sigma_{d}=+.90$ (Table 1B). Self-consistency was somewhat reduced (Figure 2E) but remained high. The net effect that emerged from the pairwise comparisons is an overestimation of the horizontal axis of the ellipses. In particular, when a circle was traced by a spot that decelerated around the 3- and 9-o'clock positions, there was an overall tendency to perceive the trajectory as a horizontal ellipse.

An explanation for this general trend can be attempted by resorting to the notion of "natural motion," which has already been put forward to account for the relationship between physical and perceived motion (Runeson, 1974). A number of studies (Cohen, 1964; Goldstein \& Wiener, 1963; Johansson, 1950a; Runeson, 1974, 1975) have shown that dynamic stimuli moving at a constant velocity are in fact perceived as negatively or positively accelerated. Conversely, certain nonuniform laws of motion produce the perceptual impression of constant velocity. These motions are qualitatively similar to those that arise in nature when a real object is acted upon by a continuing force in the presence of viscous friction (Bozzi, 1959, 1961). Thus, Runeson (1974) argues that such a realistic, but highly specific, physical model is implicitly assumed to underlie all one-dimensional motions (even when the assumption is clearly false) and summarizes the experimental findings by the general hypothesis that "only natural motions look constant" (p. 11).

Along a similar line of reasoning, we could argue that many natural motions arise from the combined action of forces acting against visco-elastic impedances and are described by second-order differential equations (Newton's Second Law). Consequently, their Cartesian components are well approximated by harmonic functions. In particular, any physical object that can be construed as a 3-D inverted pendulum under the action of nonaxial forces will exhibit elliptic oscillations that satisfy the Lissajous model. Thus, it may well be possible that, in the course of phylogenesis, the elliptic motion has become so intimately associated with the particular perceptual quality of the harmonic law of motion that, on perceiving such a dynamic form, we assume implicitly a Lissajous mode of generation. Note that the origin of this association need not be confined to perception. As mentioned in the introduction, the Lissajous model applies in particular to elliptic movements produced voluntarily with the upper limbs. Moreover, it can be shown (e.g., Hollerbach, 1980) that many common movements, such as writing, can be closely approximated by suitable generalizations of the same model. Therefore, reafferences from the motor commands could provide a continuing additional source of association be- 
tween the elliptic shape and a specific law of motion. In short, we are here suggesting the hypothesis that only Lissajous elliptic motions look natural. If so, one can assume that whenever a discrepancy exists between the perceived velocity and the one that, according to the model, would be coherent with the geometry of the trajectory, humans tend to distort geometry so as to reduce the discrepancy. The illusory flattening of the trajectories in the first test condition is in keeping with this qualitative hypothesis because, as mentioned in the introduction, the Lissajous model implies that curvature increases when velocity decreases.

The results of the second test condition do not provide direct support to the hypothesis expressed above because the population average of the differential limen is almost identical to that measured in the constant-velocity condition. However, the significant decrease of the differential sensitivity (the standard deviation increases from .189 to .236) for the entire population may be taken to suggest that discriminability is adversely affected by the conflict between the geometry of the stimulus and the expected velocity. Finally, it must be stressed that the suggested distinction between an effect related to movement per se and one that depends on the specific law of motion should not be taken to imply that the two phenomena are independent, or that they interact additively. It is clear, though, that the available data are insufficient to speculate further on the nature of their interaction.

\section{REFERENCES}

Abramowitz, M., \& Stegun, A. (1972). Handbook of mathematical functions. New York: Dover.

ADLER, B., \& Grüsser, O.-J. (1979). Apparent movement and appearance of periodic stripes during eye movements across a stroboscopically illuminated random dot pattern. Experimental Brain Research, 37, 537-550.

BehrenS, F., GrüssER, O.-J. (1978). Bewegungswahmehmung und Augenbewegungen bei Flickerbeleuchtung unbewegter visueller Muster. In G. Kommerell (Ed.), Augenbewegungsstörungen (pp. 273-284). München: J. F. Bergmann.

BENussi, V. (1912). Stroboscopische Scheinbewegungen und geometrichoptiche Gestalttäuschungen. Arkiv fur die Gesamte Psychologie, 24, 31-62.

Benussi, V. (1918). Über Scheinbewegungskonbination. Arkiv für die Gesamte Psychologie, 37, 232-282.

BörJESSON, E., \& vON HOFSTEN, C. (1972). Spatial determinants of depth perception in two-dot motion patterns. Perception \& Psychophysics, 11, 263-268.

Böruesson, E., von Hofsten, C. (1973). Visual perception of motion in depth: Application of a vector model to three-dot motion patterns. Perception \& Psychophysics, 13, 169-179.

BozzI, P. (1959). Le condizioni del movimento "naturale" lungo i piani inclinati. Rivista di Psicologia, 53, 337-352.

BozzI, P. (1961). Fenomenologia del movimento e dinamica pregalileana. Aut Aut, 64, 1-24.

Braunstein, M. L., \& Anderson, G. J. (1984). Shape and depth perception from parallel projections of three-dimensional motion. Journal of Experimental Psychology: Human Perception \& Performance, 10, 749-760.

CoHen, R. L. (1964). Problems in motor perception. Uppsala, Sweden: Lundequistska Bokhandeln.

Corre, S. (1981). The interaction between eye movements and visual illusions. In D. F. Fisher, R. A. Monty, \& J. W. Senders (Eds.), Eye movements: Cognition and visual perception (pp. 67-81). Hillsdale, NJ: Eribaum.

CoREN, S., Girgus, J. S. (1978a). Seeing is deceiving: The psychology of visual illusions. Hillsdale, NJ: Erlbaum.

COREN, S., Girgus, J. S. (1978b). Visual illusions. In R. Held, H. W. Leibowtiz, \& H.-L. Teuber (Eds.), Handbook of sensory physiology: Vol. 8. Perception (pp. 549-568). New York: Springer-Verlag.

FINGER, F. W., \& SPELT, D. K. (1947). The illustration of the horizontalvertical illusion. Journal of Experimental Psychology, 37, 243-249.

GIBson, J. J. (1966). The senses considered as perceptual systems. Boston: Houghton-Mifflin.

Gillam, B. (1971). A depth processing theory of the Poggendorff illusion. Perception \& Psychophysics, 10, 211-216.

Goldstein, J., WiENER, C. (1963). On some relations between the perception of depth and of movement. Joumal of Psychology, 55, 3-23.

Grüsser, O.-J., \& Güsser-Cornhels, U. (1973). Neuronal mechanisms of visual movement perception and some psychophysical and behavioral correlations. In R. Jung (Ed.), Handbook of sensory physiology: Vol. 7/3a. Central processing of information (pp. 333-426). New York: Springer-Verlag.

Hollerbach, J. M. (1980). An oscillatory theory of handwriting (Tech. Rep. No. AJ-TR-534), Cambridge, MA: MIT Artificial Intelligence Laboratory.

JoHansson, G. (1950a). Configurations in event perception. Stockholm: Almqvist \& Wiksell.

Johansson, G. (1950b). Configurations in the perception of velocity. Acta Psychologica, 7, 25-79.

Johansson, G. (1973). Visual perception of biological motion and a model for its analysis. Perception \& Psychophysics, 14, 201-211.

Johansson, G. (1977). Spatial constancy and motion in visual perception. In W. Epstein (Ed.), Stability and constancy in visual perception (pp. 375-419). New York: Wiley.

Johansson, G. (1978). Visual event perception. In R. Held, H. W. Leibowitz, \& H.-L. Teuber (Eds.), Handbook of sensory physiology: Vol. 8. Perception (pp. 675-711). New York: Springer-Verlag.

KORTE, A. (1915). Kinematoscopische Untersuchungen. Zeitschnift fur Psychologie, 72, 194-296.

Lacquaniti, F., Terzuolo, C., \& Viviani, P. (1983). The law relating kinematic and figural aspects of drawing movements. Acta Psychologica, 54, 115-130.

LADD, G. T., \&OODWORTH, R. S. (1911). Elements of physiological psychology. New York: Scribner.

LAMONTAGNE, C. (1973). A new experimental paradigm for the investigation of the secondary system of human visual motion perception. Perception, 2, 167-180.

Restle, F. (1979). Coding theory of the perception of motion configuration. Psychological Review, 86, 1-24.

Robinson, J. O. (1972). The psychology of visual illusion. London: Hutchinson University Library.

Runeson, S. (1974). Constant velocity-Not perceived as such. Psychological Research, 37, 3-23.

RUNEsoN, S. (1975). Visual prediction of collision with natural and nonnatural motion functions. Perception \& Psychophysics, 18, 261-266.

SLEIGHT, R. B., \& AUSTIN, T. R. (1952). The horizontal-vertical illusion in plane geometric figures. Journal of Psychology, 33, 279-287. Sleight, R. B., * Mowbray, G. H. (1951). Discriminability between geometric figures under complex conditions. Jourmal of Psychology, 31, 121-127.

ToDD, J. T. (1982). Visual information about rigid and nonrigid motion: A geometric analysis. Joumal of Experimental Psychology: Human Perception \& Performance, 8, 238-252.

VENAR, F. A. (1948). Difference thresholds for shape distortion of geometrical squares. Joumal of Psychology, 26, 461-476.

Viviani, P., Campadelit, P., Mounoud, P. (1987), Visuo-manual tracking of human bidimensional movements. Joumal of Experimental Psychology: Human Perception \& Performance, 13, 62-78.

Viviani, P., Cenzato, M. (1985). Segmentation and coupling in complex movements. Joumal of Experimental Psychology: Human Perception \& Performance, 11, 828-845. 
Viviani, P., \& Mounoud, P. (1988). Perceptuo-motor compatibility in pursuit tracking of two-dimensional movements. Manuscript submitted for publication.

Viviani, P., Terzuolo, C. (1982). Trajectory determines movement dynamics. Neuroscience, 7, 431-437.

WERTHEIMER, M. (1912). Experimentelle Studien über das Sehen von Bewegung. Zeitschrift fur Psychologie, 61, 161-265.

\section{APPENDIX}

In this appendix, we describe the analytical procedure used to compute the instantaneous tangential velocity of the spot and the polar diagrams of Figures 1 and 2. We use the following notations:

$A_{x g}, A_{y g}$ semiaxes of the geometric trajectory of the spot (geometric ellipse).

$A_{x d}, A_{y d}$ semiaxes of the Lissajous movement that defines the velocity of the spot (dynamic ellipse).

$\Sigma_{g}, \Sigma_{d} \quad$ eccentricities of geometric and dynamic ellipses.

$E(\Sigma, \Omega)$ incomplete elliptic integral of the second kind ( $\Sigma$ : modulus, $\Omega$ : phase).

$\Phi(t) \quad$ law of motion of the spot. $0 \leq \mathrm{t} \leq 2 \pi$; $d \Phi(t) / d t \geq 0 ; \Phi(0)=0, \Phi(2 \pi)=2 \pi$.

$V(t) \quad$ tangential velocity of the spot.

$Q \quad$ common perimeter of both geometric and dynamic ellipse.

$P\left(x_{g}, y_{g}\right) \quad$ spot position on the geometric ellipse.

Four cases must be distinguished:

1. $A_{x g} \geq A_{y g}, A_{x d} \geq A_{y d}$.

2. $A_{x g} \geq A_{y g}, A_{x d} \leq A_{y d}$.

3. $A_{x g} \leq A_{y g}, A_{x d} \geq A_{y d}$.

4. $A_{x g} \leq A_{y g}, A_{x d} \leq A_{y d}$.

For simplicity, we will only develop in detail Case 1. All of the others can be worked out in a similar manner.

The general parametric equation of the geometric ellipse is

$$
\begin{aligned}
& x_{g}(t)=A_{x g} \cdot \sin \Phi(t) \\
& y_{g}(t)=A_{y g} \cdot \cos \Phi(t) .
\end{aligned}
$$

According to this equation, the velocity of $P\left(x_{g}, y_{g}\right)$ is

$$
V(t)=A_{x_{g}} \cdot d \Phi(t) / d t \cdot\left[1-\Sigma_{g}^{2} \cdot \sin ^{2} \Phi(t)\right]^{1 / 2} .
$$

In order for the law of motion to satisfy the required condition on the tangential velocity, one must also have, for any $t$,

$$
V(t)=A_{x d} \cdot\left(1-\Sigma_{d}^{2} \cdot \sin ^{2} t\right)^{1 / 2} .
$$

Eliminating $V(t)$ between these two relationships, and remembering that the geometric and dynamic ellipses have the same perimeter,

$$
Q=4 \cdot A_{x g} \cdot E\left(\Sigma_{g}, \pi\right)=4 \cdot A_{x d} \cdot E\left(\Sigma_{d}, \pi\right),
$$

one obtains the following nonlinear differential equation:

$$
d \Phi(\mathrm{t}) / d t=\frac{E\left(\Sigma_{g}, \pi\right)}{E\left(\Sigma_{d}, \pi\right)} \cdot\left[\frac{1-\Sigma_{d}^{2} \cdot \sin ^{2} t}{1-\Sigma_{g}^{2} \cdot \sin ^{2} \Phi(t)}\right]^{1 / 2} .
$$

The equation is separable and can be integrated, yielding

$$
E\left(\Sigma_{d}, \pi\right) \cdot E\left[\Sigma_{g}, \Phi(t)\right]=E\left(\Sigma_{g}, \pi\right) \cdot E\left(\Sigma_{d}, t\right) .
$$

This nonlinear implicit equation can easily be solved for the desired law of motion, $\Phi$, with the help of Landen's well-known expansion of the elliptic integral (Abramowitz \& Stegun, 1972, p. 598).

For any position $P$ of the spot on the trajectory, let $\theta_{g}$ be the angle such that

$$
\operatorname{tg} \theta_{g}=x_{g} / y_{g} .
$$

Then, the coordinates of the corresponding point $V$ on the velocity polar diagram (see Figure 1) are

and

$$
\begin{aligned}
& V_{x}=A_{x g} \cdot \frac{d E\left[\Sigma_{g}, \Phi(t)\right]}{d t} \cdot \frac{x_{g}}{\left(x_{g}^{2}+y_{g}^{2}\right)^{1 / 2}} \\
& V_{y}=A_{y g} \cdot \frac{d E\left[\Sigma_{g}, \Phi(t)\right]}{d t} \cdot \frac{y_{g}}{\left(x_{g}^{2}+y_{g}^{2}\right)^{1 / 2}} .
\end{aligned}
$$

(Manuscript received October 12, 1987; revision accepted for publication March 23, 1989.) 